ECONOMÍA: TEORÍA Y PRÁCTICA • Nueva Época, número 44, enero-junio 2016, pp. 147-168, http://www.izt.uam.mx/economiatyp/ojs

\title{
Dinámicas del tipo de cambio nominal y del IPC, 1991-2014: una especificación que combina los modelos ARFIMA y GARCH*
}

\author{
Dynamics of the Nominal Exchange Rate and the IPC, \\ 1991-2014:
}

a Specification that Combines Models ARFIMA and GARCH

Héctor F. Salazar-Núñez** y Francisco Venegas-Martínez***

\section{RESUMEN}

En este trabajo se utilizan los modelos ARFIMA y GARCH, así como combinaciones de ellos para detectar algún tipo de memoria en el tipo de cambio nominal USD-MXN y el Índice de Precios y Cotizaciones de la Bolsa Mexicana de Valores durante el periodo 1991-2014. El principal hallazgo empírico es que ambas series presentan evidencia de memoria larga y de ARCH. Sin embargo, los modelos ARFIMA y GARCH no explican por sí mismos el comportamiento de las variables, mientras que su combinación (la media tiene memoria larga y la varianza cambia con el tiempo) presenta un mejor ajuste de acuerdo a las pruebas de Hosking y de Sowell y al criterio de información de Akaike.

Clasificación JEL: N2, C58.

Palabras clave: mercados bursátiles, mercados cambiarios, memoria larga, modelos econométricos de series temporales.

\footnotetext{
Abstract

This paper uses the ARFIMA and GARCH models and combinations of them to detect if some type of memory exists in the nominal exchange rate USD-MXN and in the Mexican Stock Exchange Index during the period 1991-2014. The main empirical finding is that both series present evidence of long memory and ARCH. However, the ARFIMA and GARCH models fail to explain by themselves the movements of these variables, while the combination of the methodologies (the mean has long memory and the variance changes with time) present the best fit according to Hosking y Sowell tests, and the Akaike information criterion. JEL classification: N2, C58.

Keywords: stock markets, exchange-rate markets, long memory, time series econometric models.

* Fecha de recepción: 07/12/2014. Fecha de aprobación: 15/02/2016.

** Escuela Superior de Economía del Instituto Politécnico Nacional. Correo electrónico: hector salazar313@gmail.com.

*** Escuela Superior de Economía del Instituto Politécnico Nacional. Correo electrónico: fvenegas 1111@yahoo.com.mx.
} 


\section{INTRODUCCIÓN}

En su artículo seminal, Fama (1970) define un mercado eficiente como aquel en el que los precios reflejan el total de la información. Una dinámica consistente con esta hipótesis es que la autocorrelación es cero o cercana a cero. No obstante, Ding, Granger y Engle (1993) encuentran series bursátiles en las que la autocorrelación cambia de signo y al aplicar transformaciones a la variable estudiada tienen una distribución diferente a la original, lo cual se contrapone a la hipótesis del mercado eficiente. Al respecto, Peters $(1994,1996)$ propone generalizarla mediante la hipótesis del mercado fractal, dado que la primera no siempre se cumple. Este autor también presenta ejemplos de cómo el modelo ARFIMA (autoregressive fractionally integrated moving average) de Hosking (1981) y una variante del modelo de Black y Scholes (1973), propuesta por McCulloch (1987), ${ }^{1}$ mantienen el mismo coeficiente de Hurst.

Existen también varios estudios sobre memoria larga en otras series económicas y financieras; Baillie, Bollerslev y Mikkelsen (1996) y Mills y Patterson (2009) realizan sendos resúmenes de ellos, el primero hasta 1995 y el segundo hasta 2006. A partir de 2008 se han publicado diversos artículos que estudian tipo de cambio e índices bursátiles. Por ejemplo, se tiene el trabajo de Dufrénot et al. (2008) que compara cuatro modelos para pronosticar la volatilidad del tipo de cambio en Alemania, Francia, Inglaterra, Holanda y Portugal de 1979 a 1999. Específicamente, estos autores utilizaron modelos ARFIMA, ARIMA (autoregressive integrated moving average), GARCH (generalized autoregressive conditional heteroskedasticity), y de volatilidad implícita. Así mismo, Aloy et al. (2011) estudian la paridad del poder de compra a través del tipo de cambio real en 78 países en el periodo 1970-2006 y encuentran que en la mayoría de ellos se presentó regresión a la media. Estos autores aplicaron pruebas de memoria larga, como el estimador local de Whittle de Robinson (1995), el estimador local de Whittle exacto de Shimotsu (2010) y la prueba de Perron y Qu (2010) basada en el logaritmo del periodograma, obteniendo resultados mixtos. Por último, Wang, Yu y Suo (2012) analizan los efectos en la política de tipo de cambio en China con respecto al dólar estadounidense.

Acerca de los índices de los mercados accionarios, se cuenta, por ejemplo, con la investigación de Hiremath y Kamaiah (2011), en la cual se analizan veinte índices de mercado con periodicidad mensual entre 1990 y 2000 y se en-

\footnotetext{
${ }^{1}$ La versión generalmente aceptada fue desarrollada por Hu y Oksendal (2003).
} 
cuentra memoria larga en Brasil, Chile y Estados Unidos, distinguiendo entre mercados emergentes y consolidados. Entre otros trabajos sobre índices bursátiles y sus componentes está el de Anoruo y Gil-Alana (2011), que estudia 11 índices de países africanos con frecuencia diaria en el periodo 1993-2006 y encuentra memoria larga en Egipto, Marruecos, Nigeria y Túnez. Así mismo, Bhattacharya y Bhattacharya (2012), quienes examinan diez índices de mercado con periodicidad diaria durante 2005-2011 y encuentran memoria larga en Brasil, Chile, China, Corea del Sur, Hungría, India, Malasia, México, Rusia y Taiwán. Por su parte, Danilenko (2009) estudia los índices por sector del omx Baltic en el periodo 2000-2008. Este índice incluye a los sectores de comunicaciones, energía, consumo, salud, financiero, tecnología y de servicios y dicho autor encuentra memoria larga en ellos. Asimismo, Mukherjee y Sarkar (2011) estudian el Sensex, un aproximado del índice de la India, con periodicidad diaria durante el periodo 1997-2009. Por otro lado, Saleem (2014) analiza los índices del mercado financiero de Rusia y su eficiencia. Para ello, examina los sectores de energía, bienes de consumo y telecomunicaciones con periodicidad diaria durante 20042013 y encuentra alta correlación y memoria larga en sus índices, antes y después de la crisis de 2008, obteniendo un coeficiente de Hurst significativo durante la crisis financiera. Por último, es importante mencionar que SalazarNúñez y Venegas-Martínez (2015) estudian la dinámica del tipo de cambio del dólar estadounidense con respecto a 19 economías, desarrolladas y en vías de desarrollo, incluido México, con el fin de hallar evidencia de memoria larga durante el periodo 1971-2012. Estos autores encuentran confirmación en ese sentido en los casos de Chile, China, Islandia y México.

Un tema de actualidad en investigación es si la volatilidad en los mercados accionarios y de divisas presenta efectos de largo plazo, de manera que los impactos externos que generan volatilidad en estos mercados también perduren en la dinámica futura de variables macroeconómicas fundamentales. La anticipación de posibles efectos negativos de dichos impactos en el largo plazo es esencial en el diseño y la instrumentación de la política económica. También es importante saber si las economías vecinas tienen volatilidad persistente que pudiera afectar en el largo plazo los mercados financieros locales a fin de tomar medidas precautorias para administrar el riesgo cambiario. ${ }^{2}$ Desde el trabajo de Venegas-Martí-

\footnotetext{
${ }^{2}$ Véase Venegas-Martínez, Tinoco y Torres (2009) sobre efectos de largo plazo en el mercado accionario y González-Aréchiga, Díaz y Venegas-Martínez (2001) sobre riesgo cambiario de largo plazo.
} 
nez e Islas (2005), que examina la volatilidad en los mercados accionarios de América Latina durante 1988-1998 y encuentra memoria larga en Argentina, Brasil, Chile y México, no se ha efectuado un trabajo con datos hasta 2014 para examinar si esta memoria aún subsiste en México, tanto para el mercado accionario como para el cambiario. El presente trabajo analiza el Índice de Precios y Cotizaciones (IPC) de la Bolsa Mexicana de Valores y el tipo de cambio nominal entre peso mexicano (MXN) y el dólar estadounidense (USD) en busca de algún tipo de memoria, ya sea corta o larga, para lo cual se utilizan modelos ARFIMA y GARCH. ${ }^{3}$ Las hipótesis de este trabajo de investigación son:

1) Tanto la serie del IPC como la del tipo de cambio nominal presentan memoria larga.

2) La combinación de los modelos ARFIMA y GARCH presenta un mejor ajuste que utilizados de manera independiente, es decir, la media tiene memoria larga y la varianza cambia con el tiempo.

Con el fin de validar las hipótesis, se estimará un modelo ARFIMA con base en las metodologías propuestas por Hosking (1981) y Sowell (1992), ambas en el dominio del tiempo. Asimismo, se empleará un modelo GARCH, propuesto por Bollerslev (1986), para modelar el comportamiento de las series bajo estudio cuando la varianza condicional está en función del tiempo.

La organización del presente trabajo es como sigue: en la sección i se discuten las posibles definiciones de memoria larga, se desarrollan los supuestos y modelos, y se revisan las técnicas de detección de memoria corta o larga. En la II, se realiza un análisis estadístico descriptivo de las variables IPC y tipo de cambio, se aplican las pruebas de detección de memoria y se estiman los modelos ARFIMA y GARCH y la combinación de ellos. En la última sección se presentan las conclusiones.

\section{DEFINICIÓN DE MEMORIA, MOdELOS Y PRUEBAS}

Se puede definir un proceso estacionario con memoria corta y larga si la suma de la autocorrelación tiende a ser finita o infinita, respectivamente. De esta manera,

${ }^{3}$ El modelo GARCH no es estrictamente utilizado para modelar memoria; véase, por ejemplo, Bollerslev (1986) y Ding, Granger y Engle (1993), quienes aseguran que sí se puede capturar el fenómeno de memoria, corta o larga, bajo ciertas condiciones. 
McLeod y Himpel (1978) definen que una serie temporal, $y_{t}$, tiene memoria larga si satisface

$$
\lim _{n \rightarrow \infty} \sum_{k=-n}^{k=n}\left|\rho_{k}\right|=\infty
$$

donde $\rho_{k}$ es la función de autocorrelación de $y_{t}$. El valor absoluto que aparece en (1) es para asegurar que los valores negativos se cancelen con los positivos, lo cual podría llevar a una serie convergente. Existen otras definiciones alternativas en los trabajos de Beran (1992), Taqqu, Teverovsky y Willinger (1995), Granger y Hyung (2004) y Ashley y Patterson (2010). Por ejemplo, las siguientes tres definiciones: $\operatorname{var}\left(\bar{y}_{t}\right) \approx c T^{-\alpha}$ cuando $T \rightarrow \infty ; \rho(k) \approx c k^{-\alpha}$ cuando $k \rightarrow \infty$, y $f(\lambda) \approx c \lambda^{-\beta}$ cuando $\lambda \rightarrow 0$, donde $c$ es una constante positiva, y $\alpha, \beta$ satisfacen $0<\alpha<1$ y $0<\beta<1$. La función $f(\lambda)$ es la densidad espectral. La razón por la que se toman $\alpha$ y $\beta$ con $0<\alpha<1$ y $0<\beta<1$ es para asegurar que $\rho(k)$ y $f(\lambda)$ converjan a cero cuando $k \rightarrow \infty$. Como se observa, la primera definición se refiere a la varianza, la segunda a la correlación y la tercera a la densidad espectral.

Por su parte, Granger y Joyeux (1980) y Hosking (1981) propusieron, de manera independiente, un modelo que permite capturar memoria larga. El segundo autor considera una extensión de la metodología Box-Jenkins y es el modelo más utilizado, conocido como ARFIMA. La otra clase de modelos útil para detectar memoria larga en las series temporales, de acuerdo con Bollerslev (1986) y Ding, Granger y Engle (1993), es la GARCH $(p, q)$. Dentro de la familia de estos modelos, existe uno especialmente creado para detectar memoria larga, el FIGARCH (fractionally integrated generalized autoregressive conditionally heteroskedastic), propuesto por Baillie, Bollerslev y Mikkelsen (1996), pero que queda fuera del interés del presente trabajo. ${ }^{4}$

Por otro lado, Hosking (1981) desarrolló un modelo ARIMA de memoria larga bajo dos condiciones de comportamiento: la primera de ellas es que se trata con un modelo $(0, d, 0)$ y la segunda con $p \neq 0$ y $q \neq 0$. Actualmente se le conoce como modelo ARFIMA y se representa con la siguiente ecuación:

$$
A(L)(1-L)^{d} y_{t}=B(L) \varepsilon_{t} \quad \varepsilon_{t} \sim N\left(0, \sigma_{\varepsilon}^{2}\right),
$$

donde

$$
A(L)=1-\alpha_{1} L-\cdots-\alpha_{p} L^{p}, B(L)=1-\beta_{1} L-\cdots-\beta_{q} L^{q},
$$

\footnotetext{
${ }^{4}$ Véase, también, Pavlova et al. (2014).
} 
y $L$ es el operador de rezago, mientras que $(1-L)^{d}$ es el operador de rezago fraccional y $\varepsilon_{t}$ es un proceso de ruido blanco. De esta manera, el parámetro $d$ es un número real que puede tomar valores en el intervalo cerrado $[-0.5,0.5]$. Sí $d<0.5$, entonces $y_{t}$ es estacionaria y todas las raíces de $A R(p)$ están fuera del círculo unitario. Si $d>-.05$, entonces $y_{t}$ es invertible y todas las raíces $M A(q)$ están fuera del círculo unitario. $\mathrm{Si}-0.5<d<0.5$, entonces $y_{t}$ es estacionaria e invertible, de tal manera que la función de autocorrelación, $\rho_{k}$, existe y es finita cuando $k \rightarrow \infty$. Si $-0.5>d>0.5$, entonces la varianza de $y_{t}$ es infinita y $y_{t}$ no es estacionaria. $^{5}$

El modelo GARCH, propuesto por Bollerslev (1986) como una generalización del modelo ARCH (autoregressive conditionally heteroskedastic) de Engle (1982), se utiliza principalmente para modelar el comportamiento de series temporales en las que la varianza condicional está en función del tiempo. Así, si $y_{t}=\sigma_{t} \varepsilon_{t}$, entonces

$$
\sigma_{t}^{2}=\omega+\sum_{i=1}^{q} \alpha_{i} \varepsilon_{t-1}^{2}+\sum_{i=1}^{p} \beta_{i} \sigma_{t-1}^{2}=\omega+A(L) \varepsilon_{t}^{2}+B(L) \sigma_{t}^{2}
$$

donde $p \geq 0, q>0, \omega>0, \alpha_{i} \geq 0, i=1, \ldots ., q, \beta_{i} \geq 0$ y $i=1, \ldots ., p$. Para que el proceso sea estacionario, se requiere que $\sum_{i=1}^{q} \alpha_{i}+\sum_{i=1}^{p} \beta_{i}<1$. Nelson y Cao (1992) demostraron que se puede relajar el supuesto sobre $\alpha_{i}$, permitiendo que pueda tomar valores negativos. Engle (2001) menciona que, en este caso, la varianza de largo plazo es $\sqrt{\omega /(1-\alpha-\beta)}$. Si la suma de los parámetros $\alpha$ y $\beta$ es menor a la unidad, se trata de un proceso de regresión a la varianza media y si, además, es cercana a la unidad, dicho proceso es lento.

Existen dos enfoques para estimar los modelos ARFIMA $(p, d, q)$ : en el dominio del tiempo y en el de la frecuencia. En el primero, se utiliza para la estimación de parámetros el método de máxima verosimilitud exacta de Sowell (1992) y de cuasi máxima verosimilitud de Bollerslev y Woldridge (1992), mientras que en el segundo se emplea el estimador de Whittle y métodos semiparamétricos. La estimación en el dominio del tiempo, vía el método máxima verosimilitud exacta se basa en que si $y_{t}$ se distribuye normalmente con media y varianza constante $\left(y_{t} \sim N(\mu, \Omega)\right)$, entonces la función de verosimilitud está dada por:

${ }^{5}$ Aunque Bloomfield (1985) establece que el parámetro $d$ puede tomar valores en el intervalo cerrado $[-1.0,0.5]$, en cuyo caso $y_{t}$ es invertible y todas las raíces $M A(q)$ están fuera del círculo unitario. 


$$
l\left(d, \alpha, \beta, \sigma_{\varepsilon}^{2}\right)=-\frac{T}{2} \ln (2 \pi)+\frac{1}{2} \ln |\Omega|-\frac{1}{2}\left(y_{t}-\mu\right)^{\prime} \Omega^{-1}\left(y_{t}-\mu\right),
$$

donde $\Omega$ es la matriz de varianzas-covarianzas y $\mu=X_{t} \beta$ es la media. Para obtener los parámetros del modelo, se procede a maximizar $l$ con respecto del vector $\left(d, \alpha, \beta, \sigma_{\varepsilon}^{2}\right)$, como lo indican Sowell (1992), Beran (1994) y Doornik y Ooms (2003).

El método para estimar el modelo GARCH $(p, q)$, según Bollerslev (1986), es el de máxima verosimilitud (aunque se puede utilizar el método de cuasi máxima verosimilitud) o el algoritmo de Berndt et al. (1974). Este último es una variante del método Newton-Gauss, propuesto por Brooks (2014). Por último, se menciona que para calcular los errores estandarizados se utiliza el método robusto de Bollerslev y Wooldridge (1992). En particular, el método de estimación es el de máxima verosimilitud, el cual considera la función

$$
l(\omega)=-\frac{T}{2} \ln (2 \pi)-\frac{1}{2} \ln |\Omega|-\frac{1}{2} \varepsilon^{\prime} \Omega^{-1} \varepsilon,
$$

donde $\Omega$ es la matriz de varianza-covarianza, $\varepsilon_{t}=y_{t}-X_{t} \beta$ ( $y_{t}$ es la serie de tiempo, $X_{t} \beta$ es sumedia). Si $\Omega=h_{t}$, entonces $h_{t}=Z_{t}^{\prime} \omega \quad\left(Z_{t}^{\prime}=\left(1, \varepsilon_{t-1}^{2}, \ldots, \varepsilon_{t-q}^{2}, h_{t-1}, \ldots\right.\right.$, $\left.h_{t-p}\right)$ y $\omega^{\prime}=\left(\alpha_{0}, \ldots, \alpha_{q}, \beta_{1}, \ldots, \beta_{q}\right)$. Para obtener los parámetros del modelo, se maximiza $l$ con respecto al vector $\omega$, como lo indica Bollerslev (1986). ${ }^{6}$

Existen diversas pruebas para verificar la existencia de memoria, ya sea larga o corta, en una serie temporal. Por ejemplo, las que calculan directamente el orden de integración -como es la propuesta de Geweke y Porter-Hudak (1983) o el método del periodograma, que se puede obtener directamente a través de la relación $d=H-1 / 2-\mathrm{y}$ otras, como el método del rango reescalado, propuesto por Hurst (1951). Es importante mencionar que existen otras pruebas de memoria, por ejemplo, las de Beran (1994), Taqqu, Teverovsky y Willinger (1995), Palma (2007) y Patterson (2012), entre otras. Estos trabajos también presentan resúmenes de la mayor parte de las pruebas existentes para la detección de memoria larga y modificaciones y avances de las mismas.

Ahora bien, a través del método del rango reescalado, se obtiene el coeficiente de Hurst, el cual se calcula por medio de la estimación de la siguiente ecuación mediante máxima verosimilitud:

\footnotetext{
${ }^{6}$ Se puede encontrar un desarrollo matemático de estimación del modelo GARCH $(p, d, q)$ con el método de cuasi máxima verosimilitud en Martin, Hurn y Harris (2013).
} 


$$
\ln \left(\frac{R_{T}}{S_{T}}\right)=c+H \ln (T),
$$

donde $\left(R_{T} / s_{T}\right)$ es el estadístico de rango reescalado, $R_{T}$ es el rango ajustado, $s_{T}$ es la desviacion estándar, $c$ es la constante de proporcionalidad, $T$ es el número de datos por intervalo y $H$ es el coeficiente de Hurst. Obsérvese que el coeficiente de Hurst puede tomar valores en $0 \leq H \leq 1$, de tal manera que se pueden presentar los siguientes casos:

1) Si $0<H<0.5$ (coincide con el orden de integracion $-0.5<d<0$ ), la serie temporal $y_{t}$ presenta memoria corta, es antipersistente y los valores medios en el largo plazo regresan a su condicion inicial.

2) Si $0.5<H<1.0$ (coincide con $0<d<0.5$ en el orden de integracion), entonces la serie temporal $y_{t}$ presenta memoria larga, es persistente y se espera que la media en el largo plazo nunca regrese a su condición inicial.

3) Si $H=0.5$ (coincide con $d=0$ ), es una caminata aleatoria, en la que los eventos presentes no influyen en el futuro.

Por otro lado, el método de la varianza agregada, derivado de una definición alterna de memoria larga, tiene como objetivo el estudio de los movimientos de la varianza de la serie temporal $y_{t}$ a través de $\operatorname{Var}\left(\overline{y_{t}}\right) \approx c T^{2 H-2}$, donde $c$ es una constante positiva, $T$ es el número de datos y $H$ es el coeficiente de Hurst. Esta metodología ${ }^{7}$ permite calcular el parámetro $H$ utilizando diferentes cortes de los datos $\left(y_{t}\right)$ y sus varianzas $\left(s^{2}\right)$. El número de varianzas obtenidas es $k$ (igual al número de cortes). La variable $k$ es un número entero que satisface la condición $2 \leq k \leq T / 2$, donde $T$ es el tamaño de la muestra. Posteriormente se realiza la regresión

$$
\ln s^{2}=\alpha_{0}+\alpha_{1} \ln k+\varepsilon,
$$

donde $s^{2}$ es el valor de la varianza de cada corte, $k$ es el número de cortes y $\varepsilon$ es el término de error. La pendiente de la regresión es negativa, y está dada por $\alpha_{1}=2 H-2$. Por último, al despejar $H$ de la última expresión, se obtiene el coeficiente de Hurst.

${ }^{7}$ El procedimiento se describe con detalle en Beran (1994) o en Taqqu, Teverovsky y Willinger (1995). 
La prueba de Geweke y Porter-Hudak (1983), o método del periodograma ${ }^{8}$ se describe a continuación. Considérese la ecuación

$$
\ln I\left(\omega_{k}\right)=\beta_{0}+\beta_{1} \ln \left[2 \operatorname{sen}\left(\frac{\omega_{k}}{2}\right)\right]^{2},
$$

donde

$$
I(\omega)=\frac{1}{2 \pi T}\left|\sum_{k=1}^{T} X_{k} e^{i k \omega}\right|^{2}
$$

es el periodograma, $\omega_{k}=\frac{2 \pi k}{T}$ son las frecuencias con su respectiva transformación con $\omega_{k}=(-\pi, \pi], \beta_{0}$ es la componente autónoma y $\beta_{1}$ es la pendiente. La estimación de la ecuación (8) se realiza a través del método de máxima verosimilitud, el cual proporciona el orden de integración, $-d=\beta_{1}$, y el coeficiente de Hurst $\left(H=1-\beta_{1} / 2\right)$ de la serie $y_{t} \cdot{ }^{9}$

Por su parte, Higuchi (1988) propuso un método para calcular la dimensión fractal $(d=2-H)$, la cual se refiere a un índice de irregularidad de la serie temporal. No obstante, el autor supone que el total de observación se comporta de manera similar a una de sus partes:

$$
L_{m}(k)=\frac{\left\{\sum_{i=1}^{\frac{t-m}{k}}|X(m+i k)-X(m+(i-1) k)| \frac{t-1}{\left(\frac{t-m}{k}\right) k}\right\}}{k}
$$

donde $X$ es la variable de estudio y $m, k$ y $t$ son números enteros; el primero representa el inicio de la serie y el segundo, el corte de la serie y, por ende, el avance progresivo de la serie de tal manera que satisfaga $m \leq k \leq t$. Para obtener la dimensión fractal y, en consecuencia, el coeficiente de Hurst, se realiza una estimación por máxima verosimilitud mediante la ecuación

$$
\ln L_{m}(k)=\ln \mathrm{c}-\alpha \ln (k),
$$

${ }^{8}$ El método del periodograma tiene varias versiones; Patterson (2012) realiza un resumen de éstas, al igual que Beran (1994).

${ }^{9}$ De acuerdo con Shimotsu y Phillips (2002), en la ecuación (7) se reemplaza 2 sen $\left(\omega_{k} / 2\right)$ por $2 \ln \omega_{k}$, para la estimación. 
donde $L_{m}(k)$ representa el resultado obtenido en (9) y $k$ es el número de bloques considerados.

Aunque el modelo GARCH $(p, q)$ fue propuesto en 1986, los avances en cuanto a probar la existencia del fenómeno de memoria larga son escasos. Por un lado, está la prueba del multiplicador de Lagrange (prueba ARCH) y, por el otro, el análisis de comportamiento de los residuos de la regresión, como son la curtosis, la prueba de normalidad y el correlograma al cuadrado. La prueba ARCH consiste en multiplicar el coeficiente de determinación $\left(R^{2}\right)$ de la regresión auxiliar y con los grados de libertad $(N-q)$. Así,

$$
\varepsilon_{t}^{2}=\alpha_{0}+\sum_{i=1}^{q} \alpha_{i} \varepsilon_{t-i}^{2}+v_{t}
$$

donde $\varepsilon_{t}^{2}$ son los residuos al cuadrado, $\alpha_{0}$ es una constante y $v_{t}$ son los residuos de la regresión. Al respecto, véase Johnston y DiNardo (1997).

El procedimiento para obtener el correlograma al cuadrado es el mismo que para el del correlograma normal. Por ejemplo, primero se obtienen los residuos $\varepsilon_{t}$, los cuales son estandarizados por su desviación estándar, luego son elevados al cuadrado y, posteriormente, para obtener la autocorrelacion, se aplica la siguiente fórmula:

$$
\rho_{n}=\frac{e_{t}^{2} e_{t-n}^{2}}{e_{t}^{2} e_{t}^{2}}
$$

donde $\rho_{n}$ es la autocorrelacion de orden $n$ y $e_{t}^{2}$ es el vector de residuos estandarizados. Para la autocorrelacion parcial, el primer rezago coincide con la autocorrelacion y para el segundo y los siguientes se aplica la siguiente fórmula:

$$
\left(\begin{array}{cccc}
\rho_{0} & \rho_{1} & \cdots & \rho_{n-1} \\
\rho_{1} & \rho_{0} & \cdots & \rho_{n-2} \\
\vdots & \vdots & \ddots & \vdots \\
\rho_{n-1} & \rho_{n-2} & \cdots & \rho_{0}
\end{array}\right)^{-1}\left(\begin{array}{c}
\rho_{1} \\
\rho_{2} \\
\vdots \\
\rho_{n}
\end{array}\right)=\left(\begin{array}{c}
\phi_{1} \\
\phi_{2} \\
\vdots \\
\phi_{n}
\end{array}\right),
$$

donde $\rho_{0}$ es igual a la unidad, $\rho_{1}, \ldots, \rho_{p}$ son las autocorrelaciones y $\phi_{1}, \ldots, \phi_{p}$ son las autocorrelaciones parciales. Por último, se aplican a los residuos de la regresión tanto la curtosis como la prueba de normalidad de Jarque-Bera. 


\section{ANÁLISIS EMPÍRICO}

Los datos que se analizan en este trabajo son los rendimientos del IPC de la Bolsa Mexicana de Valores y el tipo de cambio nominal MXN-USD (de aquí en adelante, $\mathrm{TCN})$. La transformación que se utilizará es la de los rendimientos logarítmicos $\left(\ln y_{t}-\ln y_{t-1}\right)$. Los datos fueron obtenidos del Banco de México (central) con periodicidad diaria entre el 11 de noviembre de-1991 y el 29 de agosto de 2014.

Cuadro 1. Estadísticos principales del IPC y el TCN, 11/11/1991-29/08/2014

\begin{tabular}{lccccccccccr}
\hline Datos & T & Media & DE & Min. & Max. & Sesgo & Curtosis & JB & ADF & KPSS $_{c}$ & KPSS $_{\text {cy }}$ \\
IPC & 5727 & 0.00 & 0.02 & -0.14 & 0.12 & 0.03 & 8.98 & 8526 & -68.14 & 0.05 & 0.03 \\
TCN & 5727 & 0.00 & 0.01 & -0.16 & 0.20 & 3.30 & 114.75 & 2990200 & -14.02 & 0.36 & 0.05 \\
\hline \hline
\end{tabular}

Nota: T: número de observaciones, DE: desviación estándar, Min.: mínimo, Max.: máximo, JB: Jarque-Bera, ADF: Dickey-Fuller aumentada, - 2.86 y - 2.57, KPSS: Kwiatkowski-Phillips-SchmidtShin, KPSS ${ }_{c}: 0.46$ y 0.35, KPSS $_{\text {CyT: }}$ para datos diarios 0.15 y 0.12 , a $5 \%$ y $10 \%$, respectivamente.

Fuente: Elaboración propia con datos del Banco de México.

En el cuadro 1 se observa que los rendimientos medios de ambas variables son cercanos a cero en el periodo de estudio. El máximo del IPC coincide con la crisis financiera asiática de 1998, particularmente en Tailandia, la cual produjo un contagio en otras economías debido a la globalización del sistema financiero internacional. En cuanto al tipo de cambio, el máximo coincide con la crisis cambiaria de México que empezó a finales de 1994 y continuó durante todo el año siguiente. El sesgo es positivo, lo cual indica que en la mayor parte del periodo las cotizaciones de ambas variables fueron al alza, aunque su distribución se concentró alrededor de los rendimientos medios; de allí, el exceso de curtosis, que es mayor a ocho en ambas variables (la referencia de la distribución normal es tres), lo cual se confirma mediante la prueba de Jarque-Bera. Se realizaron varias pruebas de estacionariedad en el sentido débil. La prueba de raíz unitaria ADF (de Dickey-Fuller aumentada) (Dickey y Fuller, 1981) muestra que ninguna de las series posee una raíz unitaria, por lo tanto, pueden ser estacionarias. Asimismo, una prueba más robusta de estacionariedad, como la KPss (Kwiatkowski-Phillips-Schmidt-Shin) (Kwiatkowski, 1992), muestra también que las dos series son estacionarias. ${ }^{10}$

\footnotetext{
${ }^{10}$ El desarrollo de las pruebas ADF y KPPSs se puede consultar en Hamilton (1994) y Enders (2008).
} 
Gráfica 1. TCN e IPC, 11/11/1991-29/08/2014
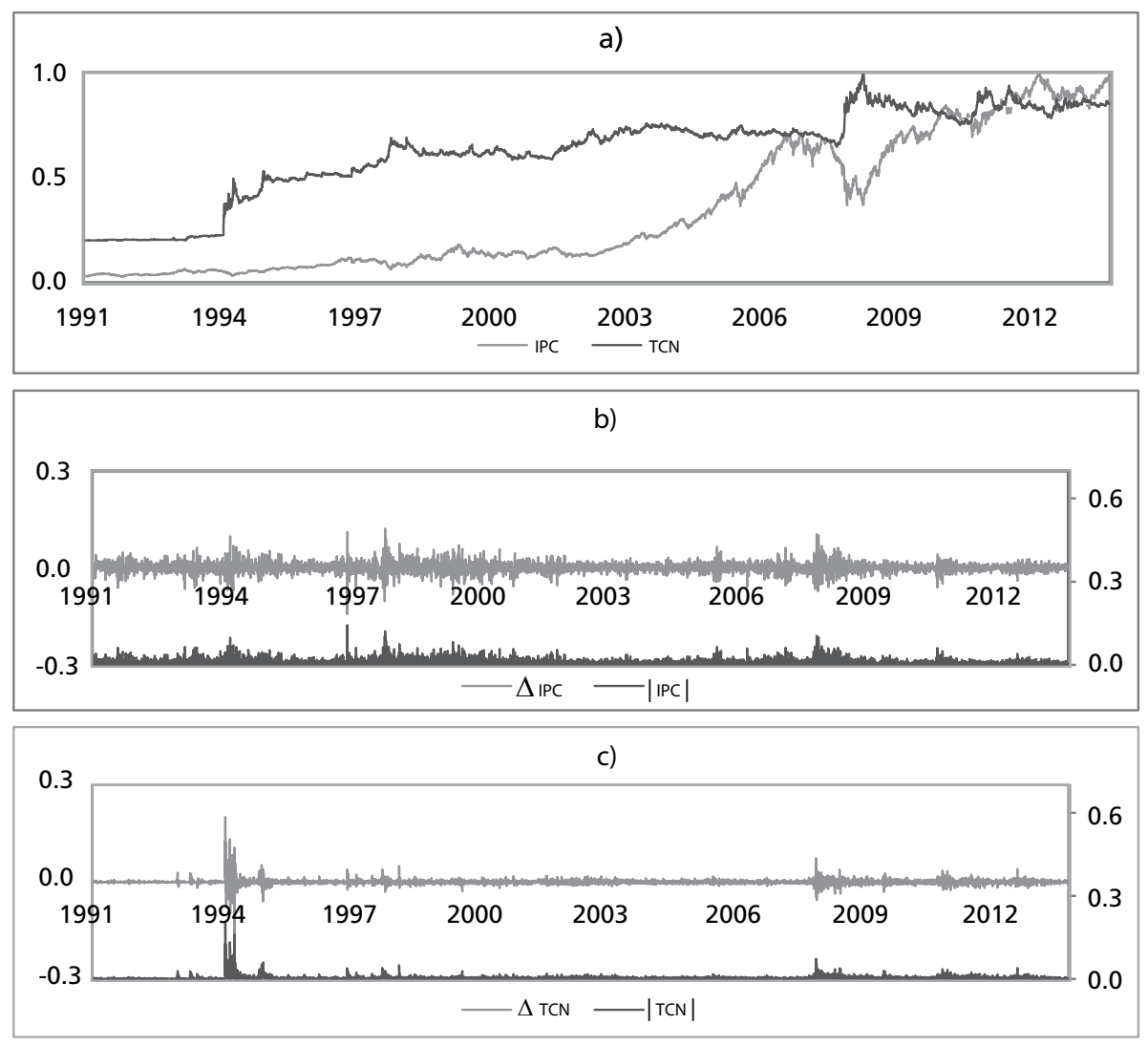

Fuente: Elaboración propia con datos del Banco de México.

En el panel a) de la gráfica 1, se puede observar que tanto el TCN como el IPC siguen trayectorias similares, con un coeficiente de correlación de $74.46 \%$, a excepción del periodo de la crisis hipotecaria de 2008-2010, durante el cual se observa que cuando hay depreciaciones del peso mexicano, dado al aumento de la cotización del dólar estadounidense (ubicándose el máximo en MXN 15.37 el 2 de abril del 2009), el índice de mercado empieza a bajar. En el panel b), se presentan los rendimientos y el valor absoluto del TCN. Se observan dos picos pronunciados: el primero en 1994-1995 y el segundo en 2008-2009, coincidiendo con la crisis cambiaria y la hipotecaria, aunque también se ven variaciones considerables desde 2011 hasta el presente. Por último, en el panel c), se observa una diferencia considerable en los movimientos del IPC, ya que éste es más volá- 
til que el TCN, tal vez porque el periodo de estudio abarca dos regímenes de cambio: el de bandas cambiarias con desliz (o banda de flotación), del 11 de noviembre de 1991 al 12 de diciembre de 1994, y la libre flotación (o flotación administrada), desde el 22 de febrero 1994 al presente (Banco de México, 2009).

Ding, Granger y Engle (1993) mencionan que si la serie $y_{t}$ sigue un proceso idénticamente distribuido, cualquier transformación que se le realice seguirá el mismo proceso. En la gráfica 2, se muestran las primeras cien autocorrelaciones del IPC y del TCN y de sus transformaciones. En este caso, se presentan el valor absoluto de los rendimientos y el cuadrado de los mismos. Las bandas (límite superior e inferior) a $95 \%$ de confianza fueron calculadas con $\pm 1.96 / \sqrt{T} \cdot{ }^{11}$

\section{Gráfica 2. Autocorrelación del IPC y del tipo de cambio}

a)

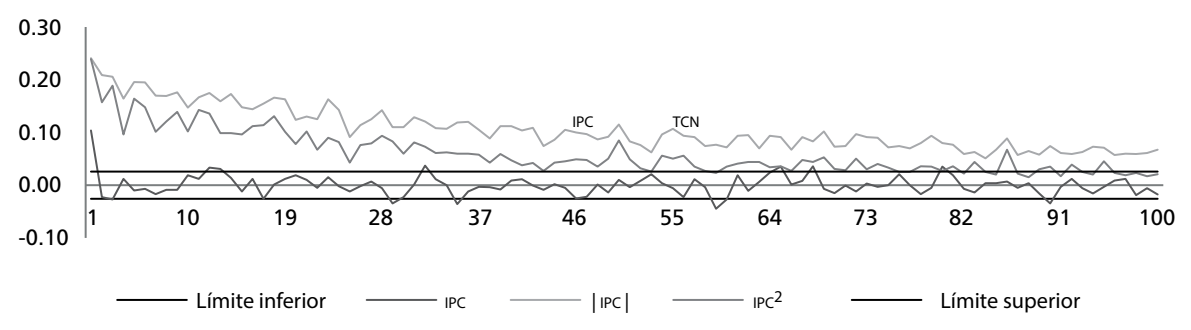

b)

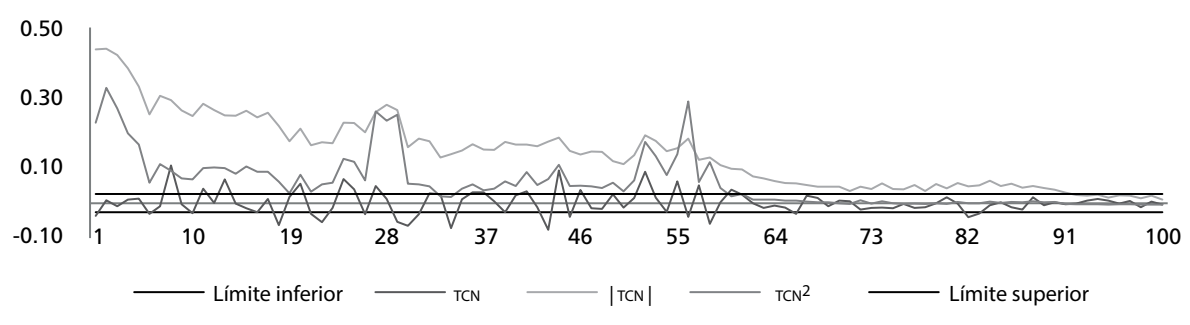

Fuente: Elaboración propia con datos del Banco de México.

En el panel a) de la gráfica 2 se observa que las primeras autocorrelaciones de los rendimientos se mueven dentro de las bandas y posteriormente empiezan a variar demasiado, saliéndose de las bandas y, finalmente, regresando y estabilizándose. Después se presenta un cambio de signo, que va de positivo a

\footnotetext{
${ }^{11}$ Algunos autores, como Beran (1994), las calculan mediante $\pm 2 / \sqrt{T}$.
} 
negativo y viceversa, parecido a un proceso que tiene regresión a la media. Los rendimientos al cuadrado tienen movimientos parecidos a la variable anterior, con la diferencia de que son más pronunciados y, por último, el valor absoluto presenta una caída lenta en las autocorrelaciones. El panel b) muestra las autocorrelaciones de los rendimientos del TCN, los valores absolutos de los rendimientos y el cuadrado de éstos. Primero se observa que los rendimientos se mueven dentro de las bandas de $95 \%$ de confianza. Después se observan cambios de signo, de positivo a negativo, característica de un proceso de memoria con regresión a la media. Por último, tanto los rendimientos absolutos como el cuadrado de ellos se mueven de manera similar, teniendo una caída lenta en las autocorrelaciones y permaneciendo fuera de las bandas de confianza.

Las autocorrelaciones de las variables transformadas del IPC y del TCN no tienen una distribución similar a la variable original, dado que no son independientes ni están idénticamente distribuidas. Se observan dos hechos: Por un lado, las autocorrelaciones de la variable original cambian de signo: de positivo a negativo, el IPC, y de negativo a positivo, el TCN. En segundo lugar, en las autocorrelaciones de los rendimientos absolutos se observa una caída lenta, al menos hasta el centésimo rezago, con lo cual se tienen dos síntomas del fenómeno de memoria larga.

Cuadro 2. Simulación de coeficiente de Hurst

\begin{tabular}{ccccccccc}
\hline$\alpha$ & $\mathrm{R} / \mathrm{s}-\mathrm{IPC}$ & $\mathrm{R} / \mathrm{s}-\mathrm{TCN}$ & VA-IPC & VA-TCN & GPH-IPC & GPH-TCN & H-IPC & H-TCN \\
\hline 0.2 & 0.68 & 0.59 & 0.85 & 0.84 & 0.81 & 0.83 & 0.97 & 0.99 \\
0.3 & 0.62 & 0.80 & 0.83 & 0.80 & 0.80 & 0.84 & 0.97 & 0.95 \\
0.4 & 0.63 & 0.76 & 0.83 & 0.81 & 0.78 & 0.84 & 0.98 & 0.98 \\
0.5 & 0.62 & 0.77 & 0.84 & 0.81 & 0.77 & 0.83 & 0.97 & 0.97 \\
0.6 & 0.62 & 0.77 & 0.83 & 0.80 & 0.77 & 0.83 & 0.97 & 0.97 \\
0.7 & 0.62 & 0.77 & 0.84 & 0.81 & 0.77 & 0.83 & 0.97 & 0.97 \\
0.8 & 0.62 & 0.77 & 0.84 & 0.81 & 0.77 & 0.83 & 0.97 & 0.97 \\
0.9 & 0.62 & 0.77 & 0.84 & 0.81 & 0.77 & 0.83 & 0.97 & 0.97 \\
\hline
\end{tabular}

Nota: R/s: rango reescalado, VA: varianza agregada, GPH: prueba de Geweke y Porter-Hudak, H: método de Higuchi.

Fuente: Elaboración propia con datos del Banco de México, utilizando el software R, paquete f-ARMA. 
En el cuadro 2 se muestra el coeficiente de Hurst calculado para diferentes valores del parámetro $\alpha$, dada la regla de un medio $\left(T^{\alpha}=m\right)$. Se puede observar que el resultado del coeficiente de memoria, en todos los casos, indica memoria larga, es decir $H>0.5$. El método del rango reescalado arroja un coeficiente menor para el IPC y un coeficiente mayor para el método de Higuchi. Las pruebas de la varianza agregada y de GPH (Geweke y Porter-Hudak) proporcionan resultados similares. Entonces, de acuerdo con estos resultados, el IPC y el TCN tienen memoria larga más o menos constante, dado que $\alpha$ toma valores desde 0.4 hasta 0.9 .

El cuadro 3 fue obtenido introduciendo las variables a un proceso de tipo Box-Jenkins, por lo cual se limpió el correlograma hasta el vigésimo rezago, ya que en esta metodología sólo los primeros rezagos tienen importancia en la explicación de la variable. De esta manera, el IPC y el TCN satisfacen los modelos tipo ARIMA. Sus resultados muestran que los residuos no tienen una distribución normal con media cero y varianza constante, dado que el sesgo en ambos casos es positivo. La forma de la campana es de tipo leptocurtica y ninguna pasa la prueba de normalidad Jarque-Bera; aunado a esto, tampoco pasan la prueba ARCH que va del primero al décimo rezago.

Cuadro 3. Estadísticos de los residuos

\begin{tabular}{|c|c|c|c|c|c|c|c|c|c|}
\hline & $T$ & Media & $\mathrm{DE}$ & Sesgo & Curtosis & $\mathrm{JB}$ & ARCH-1 & ARCH-5 & ARCH-10 \\
\hline IPC & 5725 & 0.00 & 0.02 & 0.10 & 9.19 & 9162 & 402 & 598.49 & 668.58 \\
\hline TCN & 5714 & 0.00 & 0.01 & 3.42 & 116.43 & 3074274 & 281 & 908.08 & 947.37 \\
\hline
\end{tabular}

Nota: DE: desviación estándar, JB: Jarque-Bera, ARCH: con uno, cinco y diez rezagos. Fuente: Elaboración propia utilizando el software R.

En la gráfica 3 se muestra el correlograma de los residuos al cuadrado del IPC y del TCN, los cuales fueron calculados con las ecuaciones (12) y (13). Las bandas consideran dos desviaciones estándar $\pm 1.96 / \sqrt{T}$.

Se observa en la gráfica que los residuos al cuadrado de las variables estudiadas no se distribuyen con media cero y varianza constante. Es decir, existe una caída lenta en las autocorrelaciones de los residuos al cuadrado, aunque el signo permanece. Los resultados indican que las variables pueden ser estimadas por ambas metodologías -ARFIMA $(p, d, q)$ y GARCH $(p, q)$-, dado que, por un lado, se encontró que las series tenían memoria larga (cuadro 2) y, por otro, que la varianza no era constante (cuadro 3). 
Gráfica 3. Correlograma de los residuos al cuadrado del IPC y del TCN

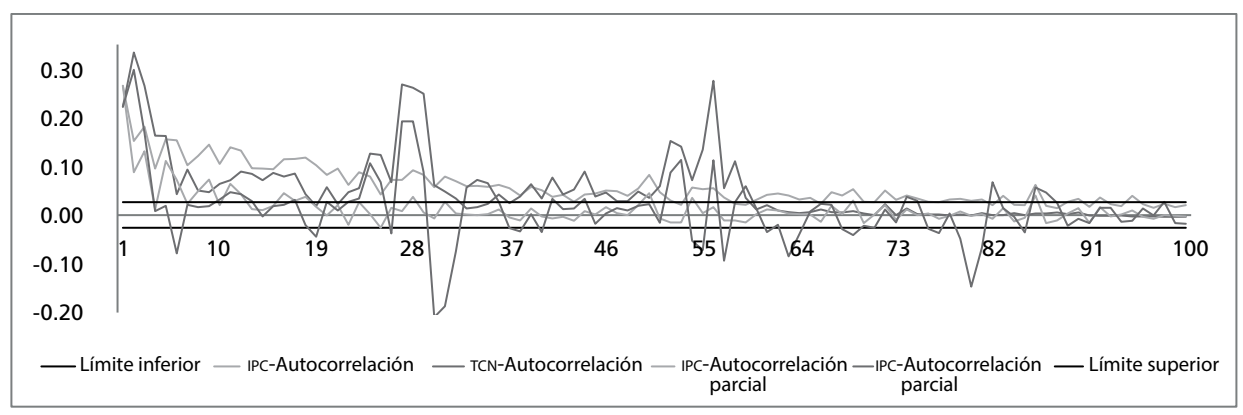

Fuente: Elaboración propia con datos del Banco de México.

Se puede observar en el cuadro 4 que el modelo combinado tiene mejor ajuste por la metodología de Hosking (1981), debido a que el criterio de Akaike es más bajo. Los parámetros del modelo GARCH son muy cercanos a la unidad, lo cual indica que la varianza tiene regresión a la media y ésta es muy lenta, al menos en el caso del índice del mercado accionario.

Cuadro 4. Estimación del modelo ARFIMA $(p, d, q)$ y $\operatorname{GARCH}(p, q)$

\begin{tabular}{|c|c|c|c|c|c|c|c|c|c|c|}
\hline & \multicolumn{2}{|c|}{ GARCH } & \multicolumn{2}{|c|}{ ARFIMA $_{1}$} & \multicolumn{2}{|c|}{ ARFIMA $_{2}$} & \multicolumn{2}{|c|}{ ARFIMA-GARCH } & \multicolumn{2}{|c|}{ ARFIMA $_{2}-\mathrm{GARCH}$} \\
\hline & IPC & TCN & IPC & $\mathrm{TCN}$ & IPC & TCN & IPC & TCN & IPC & $\mathrm{TCN}$ \\
\hline$\alpha_{0}$ & 0.00 & 0.00 & 0.00 & 0.00 & 0.00 & 0.00 & 0.00 & 0.00 & 0.00 & 0.00 \\
\hline$\alpha_{1}$ & 0.11 & 0.06 & & -0.25 & -0.21 & -0.06 & 0.00 & & 0.07 & \\
\hline$\beta_{1}$ & & & & & 0.36 & & & & & \\
\hline$d$ & & & 0.12 & 0.27 & -0.04 & 0.03 & 0.12 & 0.27 & 0.04 & 0.01 \\
\hline$\omega$ & 0.00 & 0.00 & & & & & 0.00 & 0.00 & 0.00 & 0.00 \\
\hline$\alpha_{1}$ & 0.10 & 0.12 & & & & & 0.16 & 0.27 & 0.20 & 0.13 \\
\hline$\beta_{1}$ & 0.89 & 0.81 & & & & & 0.76 & 0.67 & 0.78 & 0.80 \\
\hline LL & 16657 & 21568 & 15765 & 18924 & 15779 & 19035 & 16557 & 21381 & 16578 & 21561 \\
\hline $\mathrm{LB}_{\mathrm{a}}$ & 0.02 & 0.02 & 0.02 & 0.00 & 0.03 & 0.00 & 0.01 & 0.01 & 0.01 & 0.02 \\
\hline $\mathrm{LB}_{\mathrm{b}}$ & -0.01 & 0.00 & 0.00 & 0.03 & 0.03 & 0.06 & 0.02 & -0.01 & 0.02 & 0.02 \\
\hline AIC & -5.82 & -7.53 & -5.50 & -6.61 & -5.51 & -6.65 & -5.77 & -7.47 & -5.80 & -7.53 \\
\hline$\alpha+\beta$ & 0.99 & 0.93 & & & & & 0.91 & 0.93 & 0.98 & 0.93 \\
\hline
\end{tabular}

Nota: ARFIMA 1 : metodología de Hosking, ARFIMA ${ }_{2}$ : metodología de Sowell. LL: verosimilitud, $\mathrm{LB}_{a}$ : Ljung-Box con rezago de 12 residuos, $\mathrm{LB}_{\mathrm{b}}$ : Ljung-Box con rezago de 12 residuos al cuadrado, AIC: criterio de información de Akaike.

Fuente: Elaboración propia con el software Matrixer. 
El modelo ARFIMA estimado con la metodología de Sowell (1992) captura el ruido en los residuos, ya sean éstos lineales o al cuadrado, pues los valores del estadístico Ljung-Box se mantienen en las bandas alrededor de 0.03. Dados estos resultados, ninguno de los dos pudo modelar apropiadamente el comportamiento del IPC y el TCN para el periodo estudiado, del 11 de noviembre de 1991 al 29 de agosto de 2014, debido a que se siguieron presentando problemas de $\mathrm{ARCH}$, de ahí que se optara por combinar las dos metodologías, las cuales se basan en los mismos supuestos. Es decir, se supone que la media tiene memoria larga y la varianza cambia con el tiempo. Por lo tanto, se volvieron a estimar los modelos, como se observa en el cuadro 4, mediante la metodología de Hosking (1981) y Sowell (1992) y los problemas de ARCH fueron eliminados. Finalmente, es importante destacar las limitaciones y ventajas de los modelos ARFIMA y GARCH y de la combinación de ellos, así como de los resultados empíricos obtenidos.

Limitaciones:

1) En la estimación del modelo ARFIMA existen diferentes metodologías para determinar el coeficiente de Hurst. En las utilizadas, el orden de integración puede cambiar si se utilizan más o menos parámetros.

2) En la estimación GARCH usualmente no se pueden modelar los cambios drásticos de los errores de la regresión, lo cual da como resultado que la suma de los parámetros sea cercana a la unidad.

Ventajas:

1) Existen decenas de metodologías para calcular el coeficiente de Hurst. Las dos metodologías empleadas en este trabajo tienen pruebas robustas para el modelado de la volatilidad y sus efectos de largo plazo.

2) La combinación ARFIMA-GARCH permite modelar más apropiadamente el comportamiento de la varianza, con lo cual se obtienen mejores resultados en la descripción de la dinámica de índices bursátiles y tipos de cambio.

\section{CONCLUSIONES}

El propósito de esta investigación fue indagar qué modelo era mejor para captar la dinámica del IPC y el TCN durante el periodo de 11 de noviembre de 1991 al 29 de agosto de 2014, para lo cual se propusieron los modelos ARFIMA $(p, d, q)$ y 
$\operatorname{GARCH}(p, q)$. Para cada uno se realizaron pruebas generalmente aceptadas para capturar memoria larga o existencia de ARCH. Se utilizaron dos metodologías en el caso del modelo ARFIMA $(p, d, q)$ : la primera, propuesta por Hosking (1981), y la segunda, por Sowell (1992). Éstas se diferencian en que con la primera se utiliza el coeficiente de Hurst derivado del rango reescalado, mientras que en la segunda se suaviza la serie con este coeficiente y después se estima. No obstante, con la metodología de Sowell, el cálculo de orden de integración cambia conforme se utilizan más o menos parámetros. Al comparar los resultados de los modelos, son superiores los del GARCH $(p, q)$, aunque no logra modelar los cambios drásticos de los errores de la regresión, dando como resultado que la suma de los parámetros sea cercana a la unidad. Por esta razón, se combinaron ambas metodologías y se estimó un modelo que suavizara los rendimientos y que, a la vez, permitiera modelar el comportamiento de la varianza, lo cual tuvo mejores resultados, dado que eliminó el fenómeno ARCH. En conclusión, este tipo de variables pueden ser modeladas con modelos que suavicen la serie y, a la vez, traten la varianza como función del tiempo. Posiblemente también se pueden utilizar mo-

delos que supongan una varianza altamente persistente, como el IGARCH $(p, d, q)$ (integrated generalized autoregressive conditionally heteroskedastic) de Engle y Bollerslev (1986) o el FigARCH $(p, d, q)$ de Baillie, Bollerslev y Mikkelsen (1996), lo cual está considerado en la agenda futura de investigación.

\section{REFERENCIAS BIBLIOGRÁFICAS}

Aloy, Marcel; Boutaharb, Mohamed; Gentea, Karine, y Péguin-Feissollec, Anne (2011), "Purchasing Power Parity and the Long Memory Properties of Real Exchange Rates: Does One Size Fit All?", Economic Modelling, 28 (3), pp. 1279-1290.

Anoruo, Emmanuel, y Gil-Alana, Luis (2011), "Mean Reversion and Long Memory in African Stock Market Prices", Journal of Economics and Finance, 35 (3), pp. 296-308.

Ashley, Richard, y Patterson, Douglas (2010), “Apparent Long Memory in Time Series as an Artifact of a Time-Varying Mean: Considering Alternatives to the Fractionally Integrated Model”, Macroeconomic Dynamics, 14 (s1), pp. 59-87.

Baillie, Richard; Bollerslev, Tim, y Mikkelsen, Hans Ole (1996), "Fractionally Integrated Generalized Autoregressive Conditional Heteroskedasticity", Journal of Econometrics, 74 (1), pp. 3-30.

Banco de México (2009), Regímenes cambiarios de México a partir de 1954. [PDF] Consultado el 13 de noviembre de 2014, en: www.banxico.org.mx/sistema-financie- 
ro/materialeducativo/basico/\%7B51CCA803-9DB0-9162-1CFA-B19CE 71599DB\%7D.pdf.

Beran, Jan (1992), "Statistical Methods for Data with Long-Range Dependence", Statistical Science, 7 (4), pp. 404-416.

_ (1994), Statistics for long-memory processes. Vol. 61, New York, Chapman and Hall/CRC.

Berndt, Ernst; Hall, Bronwyn, Hall, Robert, y Hausman, Jerry (1974), "Estimation and Inference in Nonlinear Structural Models", Annals of Economic and Social Measurement, 3 (4), pp. 103-116.

Bhattacharya, Sharad Nath, y Bhattacharya, Mousumi (2012) "Long Memory in Stock Returns: A Study of Emerging Markets", Iranian Journal of Management Studies, 5 (2), pp. 67-88.

Black, Fischer, y Scholes, Myron (1973), "The Pricing of Options and Corporate Liabilities”, The Journal of Political Economy, 81 (3), pp. 637-654.

Bloomfield, Peter (1985), “On Series Representations for Linear Predictors”, The Annals of Probability, 13 (1), pp. 226-233.

Bollerslev, Tim (1986). "Generalized Autoregressive Conditional Heteroskedasticity", Journal of Econometrics, 31 (3), pp. 307-327.

Bollerslev, Tim, y Wooldridge, Jeffrey (1992), "Quasi-Maximum Likelihood Estimation and Inference in Dynamic Models with Time-Varying Covariances", Econometric Reviews, 11 (2), pp. 143-172.

Brooks, Chris (2014), Introductory Econometrics for Finance, Cambridge, Cambridge University Press.

Danilenko, Svetlana (2009), "Long-Term Memory Effect in Stock Prices Analysis”, Economics and Management, 0 (14), pp. 151-156.

Ding, Zhuanxin; Granger, Clive, y Engle, Robert (1993), “A Long Memory Property of Stock Market Returns and A New Model”, Journal of Empirical Finance, 1 (1), pp. 83-106.

Dickey, David, y Fuller, Wayne (1981), "Likelihood Ratio Statistics for Autoregressive Time Series with A Unit Roo", Econometrica, 49, 4, pp. 1057-1072.

Doornik, Jurgen, y Ooms, Marius (2003), Computational Aspects of Maximum Likelihood Estimation of Autoregressive Fractionally Integrated Moving Average Models, Computational Statistics and Data Analysis, 42 (3), pp. 333-348.

Dufrénot, Gilles; Lardicb, Sandrine; Mathieuc, Laurent; Mignonb, Valérie, y PéguinFeissollee, Anne (2008), "Explaining the European Exchange Rates Deviations: Long Memory or Non-Linear Adjustment?", Journal of International Financial Markets, Institutions and Money, 18 (3), pp. 207-215. 
Enders, Walter (2008), Applied econometric time series, New York, John Wiley and Sons.

Engle, Robert (1982), “Autoregressive Conditional Heteroscedasticity with Estimates of the Variance of United Kingdom Inflation”, Econometrica, 50 (4), pp. 987-1007.

- (2001), "GARCH 101: The Use of ARCH/GARCH Models in Applied Econometrics, Journal of Economic Perspectives, 15 (4), pp. 157-168.

Engle, Robert, y Bollerslev, Tim (1986), "Modelling the Persistence of Conditional Variances", Econometric Reviews, 5 (1), pp. 1-50.

Fama, Eugene (1970), "Efficient Capital Markets: A Review of Theory and Empirical Work", The Journal of Finance, 25 (2), pp. 383-417.

Geweke, John, y Porter-Hudak, Susan (1983), The Estimation and Application of Long Memory Time Series Models, Journal of Time Series Analysis, 4 (4), pp. 221-238.

González-Aréchiga, Bernardo; Díaz, Jaime, y Venegas-Martínez, Francisco (2001), "Riesgo cambiario, brecha de madurez y cobertura con futuros: análisis local y de valor en riesgo", Economía Mexicana, nueva época, 10 (2), pp. 259-290.

Granger, Clive, y Hyung, Namwon (2004), “Occasional Structural Breaks and Long Memory with an Application to The S\&P 500 Absolute Stock Returns", Journal of Empirical Finance, 11 (3), pp. 399-421.

Granger, Clive, y Joyeux, Roselyne (1980), “An Introduction to Long Memory Time Series Models and Fractional Differencing”, Journal of Time Series Analysis, 1 (1), pp. 15-29.

Hamilton, James (1994), Time Series Analysis, vol. 2, Princeton, Princeton University Press.

Higuchi, Tomoyuki (1988), “Approach to an Irregular Time Series on The Basis of the Fractal Theory”, Physica D: Nonlinear Phenomena, 31 (2), pp. 277-283.

Hiremath, Gourishankar, y Kamaiah, Bandi (2011), "Testing Long Memory in Stock Returns of Emerging Market: Some Further Evidence”, Economics, Management and Financial Markets, 6 (3), pp. 136-147.

Hosking, Jonathan (1981), "Fractional Differencing”, Biometrika, 68 (1), pp. 165-176.

$\mathrm{Hu}$, Yaozhong, y Oksendal, Bernt (2003), "Fractional White Noise Calculus and Applications to Finance. Infinite Dimensional Analysis", Quantum Probability and Related Topics, 6 (1), pp. 1-32.

Hurst, Harold Edwin (1951), "Long Term Storage Capacity of Reservoirs", Transactions of the American Society of Civil Engineers, 116, pp. 770-799.

Johnston, Jack, y DiNardo, John (1997), Econometric methods, Singapur, McGrawHill International.

Kwiatkowski, Denis (1992), “Testing The Null Hypothesis of Stationary Against the Al- 
ternative of A Unit Root: How Sure Are We That Economic Time Series Have a Unit Root?", Journal of Econometrics, 54 (1), pp. 159-178.

Martin, Vance; Hurn, Stan, y Harris, David (2012), Econometric Modelling with Time Series: Specification, Estimation and Testing, Cambridge, Cambridge University Press.

McCulloch, Huston (1987), "Foreign Exchange Option Pricing with Log-Stable Uncertainty", en A. Ghosh y S. J. Khoury (eds.), Recent Developments in International Banking and Finance, s.l., Lexington Books, p. 231

McLeod, Angus Ian, y Himpel, Keith William (1978), "Preservation of the Rescaled Adjusted Range, 1: A Reassessment of the Hurst Phenomenon", Water Resources Research, 14 (3), pp. 491-508.

Mills, Terence, y Patterson, Kerry (eds.) (2009), Palgrave Handbook of Econometrics, s.l., Palgrave Macmillan.

Mukherjee, Sen, y Sarkar, Amitava (2011), "Long Memory in Stock Returns: Insights from the Indian Market", International Journal of Applied Economics and Finance, 5 (1), pp. 62-74.

Nelson, Daniel, y Cao, Charles (1992), "Inequality Constraints in the Univariate GARCH Model, Journal of Business and Economic Statistics, 10 (2), pp. 229-235.

Palma, Wilfredo (2007), Long-Memory Time Series: Theory and Methods, New Jersey, John Wiley and Sons.

Patterson, Kerry (2012), Unit Root Tests in Time Series. Volume 2: Extensions and Developments, s.l., Palgrave Macmillan.

Pavlova, Ivelina; Cho, Jang Hyung; Parhizgari, Ali, y Hardin, William (2014), "Long Memory in REIT Volatility and Changes in the Unconditional Mean: A Modified FIGARCH Approach", Journal of Property Research, 31 (4), pp. 315-332.

Perron, Pierre, y Qu, Zhongjun (2010), "Long-Memory and Level Shifts in the Volatility of Stock Market Return Indices", Journal of Business and Economic Statistics, 28 (2), pp. 275-290.

Peters, Edgar (1994), Fractal Market Analysis: Applying Chaos Theory to Investment and Economics, New York, Wiley.

- (1996), Chaos and Order in the Capital Markets: A New View of Cycles, Prices, and Market Volatility, vol. 1, New Jersey, John Wiley and Sons.

Robinson, Peter (1995), "Gaussian Semiparametric Estimation of Long Range Dependence", The Annals of Statistics, 23 (5), pp. 1630-1661.

Salazar-Núñez, Héctor, y Venegas-Martínez, Francisco (2015), "Memoria larga en el tipo de cambio nominal: evidencia internacional (1971-2012)", Contaduría y Administración, 60 (3), pp. 615-630. 
Saleem, Kashif (2014), "Modeling Long Memory in the Russian Stock Market: Evidence from Major Sectoral Indices”, Journal of Applied Business Research, 30 (2), pp. 567-574.

Shimotsu, Katsumi (2010), "Exact Local Whittle Estimation of Fractional Integration with Unknown Mean and Time Trend", Econometric Theory, 26 (2), pp. 501-543.

Shimotsu, Katsumi, y Phillips, Peter (2002), "Poled log Periodograma Regression", Journal of Time Series Analysis, 23 (1), pp. 57-93.

Sowell, Fallaw (1992), Maximum Likelihood Estimation of Stationary Univariate Fractionally Integrated Time Series Models", Journal of Econometrics, 53 (1), pp. 165-188.

Taqqu, Murad; Teverovsky, Vadim, y Willinger, Walter (1995), "Estimators for LongRange Dependence: An Empirical Study”, Fractals, 3 (4), pp. 785-798.

Venegas-Martínez, Francisco, e Islas, Alejandro (2005), "Volatilidad en los mercados de América Latina", Comercio Exterior, 55 (11), pp. 936-947.

Venegas-Martínez, Francisco; Tinoco, Miguel Ángel, y Torres, Víctor Hugo (2009), "Desregulación financiera, desarrollo del sistema financiero y crecimiento económico en México: efectos de largo plazo y causalidad", Estudios Económicos, 24 (48), pp. 249-283.

Wang, Dong-Hua; Yu, Xiao-Wen, y Suo, Yuan-Yuan (2012), "Statistical Properties of the Yuan Exchange Rate Index", Physica A: Statistical Mechanics and its Applications, 391 (12), pp. 3503-3512. 